\title{
The place of inferior vena cava diameter and proBNP levels in determining the fluid balance of medical intensive care patients
}

\author{
Huseyin ARIKAN (D), Sait KARAKURT (1D) \\ Department of Pulmonary and Critical Care Medicine, School of Medicine, Marmara University, Istanbul, Turkey.
}

Corresponding Author: Huseyin ARIKAN

E-mail: arikanhuseyin@gmail.com

Submitted: 23.08.2020 Accepted: 17.10.2020

\begin{abstract}
Objective: Fast and accurate detection of fluid balance in intensive care patients is of special significance. Many different methods are used to determine the fluid balance. In this study, we aimed to determine the fluid balance of patients in the medical intensive care unit using measurement of the inferior vena cava diameter and pro-brain natriuretic peptide (proBNP).

Patients and Methods: Patients admitted to the medical intensive care unit between September 2013 and February 2014 were enrolled in the study. Inferior vena cava diameter was measured with a portable ultrasonography, according to guidelines published by the American Echocardiography Association. For pro BNP measurement, samples were taken simultaneously with ultrasonography. Results: One hundred and twenty-six patients (70 male,55.6\%) were enrolled for the study. Mean age was $57.8 \pm 19.8$ (18-89 years). Ninety-six (76.2\%) patients were receiving mechanical ventilation support. Mean proBNP levels were $10645.88 \pm 12731.08 \mathrm{pg} / \mathrm{ml}$. There was no statistically significant difference between proBNP levels in patients according to the fluid status. Collapsibility index was not statistically different according to the volume status $(\mathrm{p}=0.75)$.

Conclusion: proBNP levels were not correlated with the fluid balance. proBNP levels and inferior vena cava diameters were negatively but weakly correlated.

Keywords: Fluid balance, Inferior vena cava diameter, proBNP, Ultrasonography
\end{abstract}

\section{INTRODUCTION}

Assessment of fluid balance is one of the most important management procedures for critical care patients. Previously, the Swan-Ganz catheter has been used to assess the volume status of the patients; however, it lost popularity due to its invasiveness [1]. The surviving sepsis campaign guidelines in 2012 recommend using central venous pressure (CVP), but it is not recommended anymore in Sepsis 3 because of its poor correlation to the volume status of the patients [2].

In $t$ recent years non-invasive assessment is becoming popular in critical care settings. Respiratory variations in the inferior vena cava measured by ultrasonography is one of them [3]. Ultrasound is quite simple, and it can be evaluated by medical or non-medical staff following short-term instructions [4]. Also, ultrasound provides results simultaneously with the procedure. Additionally, inferior vena cava ultrasonography is well known as a tool of point-of-care for restrictive fluid therapy in heart failure or fluid removal in hemodialysis [5].

Brain Natriuretic Peptide (BNP) is a hormone whose active form is C-Terminal BNP with 32 amino acids, which is released as a 108 amino acid prohormone due to excessive stress in the heart muscle cells. The 76 amino acid N-Terminal proBNP is released in proteolysis, in which the active state of the hormone occurs [6]. It is used to evaluate and monitor heart failure. The place of BNP in fluid therapy in the intensive care unit is still a matter of debate.

In this study, we aimed to determine the fluid status of patients in the medical intensive care unit using measurement of the inferior vena cava diameter and proBNP.

How to cite this article: Arikan H, Karakurt S. The place of inferior vena cava diameter and proBNP levels in determining the fluid balance of medical intensive care patients. Marmara Med J 2021; 34(1):1-5. doi: 10.5472/marumj.858649 


\section{PATIENTS and METHODS}

The study is an observational descriptive study. We included all patients hospitalized in the Medical Intensive Care Unit (ICU) of the Marmara University Education and Research Hospital between September 2013 and February 2014 who provided an informed consent and were over 18 years of age after the approval of the Institutional Ethics Committee of Marmara University School of Medicine (Approval number: 09.2013. 0262). Patients' demographic characteristics, comorbidities, medication they were taking, vital signs, mechanical ventilator parameters and fluid status as measured by intake-output follow-ups were recorded.

Ultrasonography was performed by a certified researcher on intensive care ultrasonography, using a Mindray M5 brand portable ultrasonography device according to the guidelines of the American Echocardiography Association [7]. The right atrium was detected with a subcostal approach, the inferior vena cava was imaged, and measurements were made using M-Mode $1 \mathrm{~cm}$ above the joining point of the hepatic vein. By recording the diameters in inspiration and expiration, collapsibility and distensibility indexes were calculated as previously described in the literature [8]. For ProBNP, samples were taken simultaneously with ultrasonography and measurements were made by enzyme immunoassay method [9].

Primary end point was to determine the association of the inferior vena cava diameter indices, proBNP levels and the fluid status of patients.

\section{Statistical Analysis}

All statistical analyses were made using the PSPP program (free software). Data are given as mean \pm standard deviation and frequencies when appropriate. The Kolmogorov-Smirnov test was used to evaluate whether the distribution of data was normal. Correlation analysis was performed for the relationship between the diameter of the inferior vena cava and proBNP and the follow-up. Since the data in the subgroups were not normally distributed, the Mann-Whitney U test, one of the nonparametric tests, was used. The Kruskall-Wallis test was used when the means of more than two groups were compared. In all analyses, $\mathrm{p}<0.05$ value was taken as the limit for statistical significance.

\section{RESULTS}

Between September 2013 and February 2014, 126 patients (70 males, $55.6 \%)$ with a mean age of $57.8 \pm 19.8$ (18-89 years) were included in the study. Ninety-six (76.2\%) patients were receiving mechanical ventilatory support. Sixty-three (65.6\%) of these were on invasive, $33(34.4 \%)$ were on non-invasive ventilation. Thirty-six $(37.5 \%)$ of the patients who received mechanical ventilator support were in pressure support ventilation (PSV) mode, 23 (24\%) in pressure controlled synchronized intermittent mandatory ventilation (P-SIMV), and 36 (37.5\%) in adaptive support ventilation (ASV) mode. Average external positive end expiratory pressure (PEEP) was calculated as $5.4 \pm$
$0.9 \mathrm{mmHg}$. The demographic and clinical characteristics of the patients included in the study are summarized in Table I.

Table I. Demographic and clinical characteristics of the patients included in the study

\begin{tabular}{|c|c|}
\hline Parameters & Value \\
\hline Number of patients & 126 \\
\hline \multicolumn{2}{|l|}{ Demographics } \\
\hline Male gender, $\mathrm{n}(\%)$ & $70(55.6 \%)$ \\
\hline Age (years) & $57.8 \pm 19.8$ \\
\hline Age (years), median (min-max) & $62(18-89)$ \\
\hline Mechanical ventilation support, $\mathrm{n}(\%)$ & $96(76 \%)$ \\
\hline IMV support, $\mathrm{n}(\%)$ & $63(50 \%)$ \\
\hline NIV support, $\mathrm{n}(\%)$ & $33(26.1 \%)$ \\
\hline External PEEP $\left(\mathrm{cmH}_{2} \mathrm{O}\right)$ & $5.4 \pm 0,9$ \\
\hline \multicolumn{2}{|l|}{ Modes of mechanical ventilation, $\mathrm{n}(\%)$} \\
\hline PSV, $\mathrm{n}(\%)$ & $36(37.5 \%)$ \\
\hline P-SIMV, n (\%) & $23(24 \%)$ \\
\hline ASV, n (\%) & $36(37.5 \%)$ \\
\hline CMV, n (\%) & $1(\% 1)$ \\
\hline APACHE II & $24.3 \pm 8.1$ \\
\hline Fluid balance (ml) & $+635.8 \pm 933.8$ \\
\hline $\operatorname{proBNP}(\mathrm{pg} / \mathrm{ml})$ & $10645.9 \pm 12731.1$ \\
\hline Ejection fraction (\%) (95\% CI) & $48.4 \pm 3.6(44.1-52.8)$ \\
\hline
\end{tabular}

IMV: invasive mechanical ventilation, NIV: noninvasive mechanical ventilation, PEEP: positive end expiratory pressure, PSV: pressure support ventilation, P-SIMV: Pressure targeted synchronized intermittent mandatory mechanical ventilation, ASV: Adaptive support ventilation, CMV: controlled mechanical ventilation, 95\% CI: 95\% Confidence interval

According to input-output follow-ups (IOF), the average fluid balance of the patients included in the study was found to be $+635.8 \pm 993.8 \mathrm{ml}$. When the patients were grouped according to IOF, $26(20.6 \%)$ patients were found to be in negative fluid balance and $100(79.4 \%)$ patients were found to be in positive fluid balance. The mean fluid balance of patients with positive fluid balance according to IOF was calculated as $+958.9 \pm 742.8$ $\mathrm{ml}$, and the mean fluid balance of those with negative fluid balance was calculated as $-582.2 \pm 443.8 \mathrm{ml}$. Although the proBNP level increased in the group with positive fluid balance, there was no statistically significant difference between both groups (11705 vs 6362 respectively, $\mathrm{p}=0.153$ ). In the subgroup analysis performed excluding patients with systolic dysfunction (EF <55\%) on echocardiography, proBNP levels tended to increase as the fluid balance shifted to the positive side, but no statistically significant difference was found ( $\mathrm{p}=0.256$ ).

The mean end-inspiratory inferior vena cava diameter was $1.23 \pm 0.81 \mathrm{~cm}$, and the mean end-expiratory inferior vena cava diameter was $1.76 \pm 0.65 \mathrm{~cm}$. The best limit value for the collapsibility index to show the fluid balance was shown as $50 \%$ in previous studies. When the fluid balance averages of both groups were compared, the fluid balance of those with a collapsibility index over $50 \%$ tended to increase, but no statistically significant difference was found. However, proBNP levels were found to be higher in patients with collapsibility index less than 50\% (Table II). A statistically significant negative 
correlation was found between the calculated collapsibility indexes of the patients and the measured proBNP levels $(r=-$ $0.409 \mathrm{p}<0.01$ ); as the proBNP levels increase, the collapsibility index decreases (Figure 1).

Table II. proBNP levels according to the collapsibility index

\begin{tabular}{|l|l|}
\hline Collapsibility index $(\mathbf{n})$ & proBNP $(\mathrm{pg} / \mathrm{ml})$, mean \pm SD $(95 \%$ CI $)$ \\
\hline$<50 \%(82)$ & $12938.8 \pm 13549.9$ \\
& $(95 \%$ CI: $9658-16218,5)$ \\
\hline$\geq 50 \%(44)$ & $5939.6 \pm 9504.9$ \\
& $(95 \%$ CI: $2623.2-9256)$ \\
\hline
\end{tabular}

SD: standard deviation, CI: 95\% Confidence interval

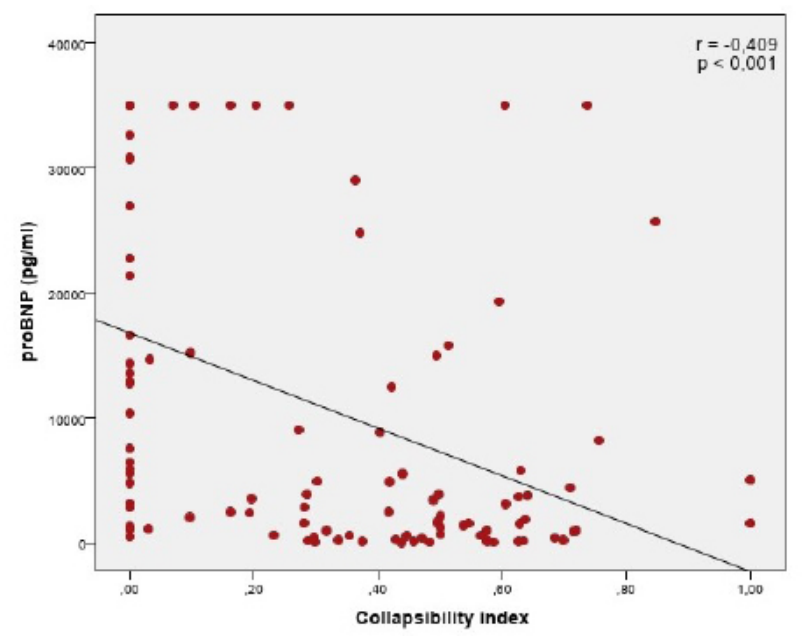

Figure 1. Collapsibility index and proBNP relationship

\section{DISCUSSION}

In our study, the place of the inferior vena cava diameter measured ultrasonographically and the collapsibility index derived from it and the proBNP level were investigated in determining the fluid balance in patients in the medical ICU. According to the results, as the positivity in fluid balance increased, proBNP levels tended to increase and the collapsibility index decreased, but the changes found did not reach statistical significance. However, a negative, weak but statistically significant relationship was found between the diameter of the inferior vena cava and proBNP level in determining the fluid balance.

In our study, although, proBNP levels tend to increase as the fluid balance shifts to the positive side, we could not show a significant relationship between proBNP levels and fluid balance. The use of proBNP in evaluating the fluid balance is mostly used in dialysis patients. In a study evaluating the hydration status of hemodialysis patients, proBNP was used to assess fluid status in relation to the diameter of the inferior vena cava [10]. In a study evaluating bioimpedance and proBNP level in peritoneal dialysis patients, it was stated that proBNP levels could be used to show fluid status such as bioimpedance and guide treatment [11]. Tapolyai et al., concluded that there was an exponential relationship between bioimpedance measurements and proBNP levels in dialysis patients [12].

The qualitative assessment of inferior vena cava diameter indices has also been carried out in a prospective study which demonstrated that they offer a rapid, non-invasive way to evaluate volume status in critically ill patients [13]. However, in our study we were unable to demonstrate similar results. Two meta-analyses showed that inferior vena cava ultrasonography is a reliable parameter for hypovolemia and has a great value in predicting fluid responsiveness $[14,15]$. However, other metaanalyses on inferior vena cava ultrasonography concluded that it is not a reliable method to predict fluid responsiveness $[16,17]$. Hence, the effectiveness of inferior vena cava ultrasonography to predict volume status or fluid responsiveness has not yet reached a conclusion.

In studies evaluating the fluid status of intensive care patients in the literature, the inferior vena diameter was mostly compared with CVP. Zhang et al., in their study of 32 patients who had undergone gastrointestinal surgery, the fluid status of the patients was evaluated with CVP and the inferior vena cava diameters were evaluated by a researcher who was blind to the results. As a result, a correlation was found between CVP measurements and inferior vena cava diameters $(r=0.585, \mathrm{p}<0.05)$ [18]. In this study, as only a very small number of patients had central lines to evaluate CVP (eight patients) we were unable to make such a comparison.

In our study, we found a negative, weak but statistically significant relationship between inferior vena cava diameters and proBNP levels. In addition, when we divided the patients into two groups with a $50 \%$ limit value of the collapsibility index, we found a statistically significant difference between the groups in favor of those with a collapsibility index of less than $50 \%$. The reason for using the $50 \%$ limit is that it was reported as the most successful limit value in demonstrating fluid balance in previous studies [19]. This finding shows that the collapsibility index and proBNP levels are in agreement with each other in determining the fluid balance.

According to our knowledge, there is no study in the literature evaluating proBNP levels and inferior vena cava diameter in medical intensive care patients. Current studies have been conducted in heart failure patients. In a study investigating the correlation of the inferior vena cava diameter and natriuretic peptides in heart failure patients, a positive but weak relationship was found ( $r=0.27,95 \% \mathrm{CI}: 0.05-0.47 ; \mathrm{P}=0.01$ ) [20]. In a study evaluating the re-admissions of patients hospitalized with the diagnosis of acute decompensated heart failure after discharge, it was reported that patients with high proBNP levels at discharge and with larger inferior vena cava diameters at presentation had earlier and more frequent hospitalizations [21]. In a study conducted in our country, the treatment responses of patients admitted to the emergency department with acute heart failure were evaluated with the inferior vena cava collapsibility index and there was a statistically significant difference between 
pre-treatment collapsibility indexes and post-treatment collapsibility index $(\mathrm{p}<0.001)$, but there was no difference between proBNP levels [22].

\section{Limitations}

The limitations of this study should be acknowledged. First, invasive assessments of fluid status were very limited in the current study. Thus, comparisons between invasive methods and inferior vena cava ultrasonography could not be made. However, the correlation between invasive techniques and inferior vena cava ultrasonography has already been shown in previous studies as mentioned earlier. Secondly, there was no consecutive measurement during or after fluid therapy. Therefore, no comment could be made on changes in the indeces.

\section{Conclusion}

In conclusion, as the positivity in fluid balance increases, proBNP levels tend to increase, and the collapsibility index tends to decrease. In addition, a weak negative but statistically significant relationship was found between the proBNP level and the collapsibility index, and it was shown that these two parameters were compatible with each other in evaluating the fluid balance.

\section{Compliance with Ethical Standards}

Ethical Approval: The study protocol was approved by the Institutional Ethics Committee of Marmara University School of Medicine (Approval number: 09.2013. 0262). All patients provided written informed consent.

Financial Support: The authors have no relevant financial information to disclose

Conflict of Interest: The authors have no potential conflicts to declare

Authors Contributions: Initials of the contributing authors are listed in brackets at the relevant parts of the research: Literature search (H.A.), Study design (H.A., S.K.), Legislative applications (H.A., S.K.), Data collection (H.A.), Supervision and quality control (S.K.), Statistical advice (S.K.), Statistical data analysis (H.A.), Data interpretation (H.A., S.K.), Drafting the manuscript (H.A., S.K.). Both authors approved the final version of the article.

\section{REFERENCES}

[1] De Backer D, Vincent JL. The pulmonary artery catheter: is it still alive? Curr Opin Crit Care 2018;24:204-8. doi: 10.1097/ mcc.000.000.0000000502

[2] Singer M, Deutschman CS, Seymour CW, et al. The Third International Consensus Definitions for Sepsis and Septic Shock (Sepsis-3). JAMA 2016;315:801-10. doi: 10.1001/ jama.2016.0287

[3] Theerawit P, Morasert T, Sutherasan Y. Inferior vena cava diameter variation compared with pulse pressure variation as predictors of fluid responsiveness in patients with sepsis. J Crit Care 2016;36:246-51. doi: 10.1016/j.jcrc.2016.07.023
[4] Steinwandel U, Gibson N, Towell A, Rippey JJR, Rosman J. Can a renal nurse assess fluid status using ultrasound on the inferior vena cava? A cross-sectional interrater study. International Symposium on Home Hemodialysis. Hemodial Int 2018;22:261-9. doi:10.1111/hdi.12606

[5] Alexiadis G, Panagoutsos S, Roumeliotis S, et al. Comparison of multiple fluid status assessment methods in patients on chronic hemodialysis. Int Urol Nephrol 2017;49:525-32. 10.1007/s11255.016.1473-y

[6] McLean AS, Huang SJ. The applications of B-type natriuretic peptide measurement in the intensive care unit. Curr Opin Crit Care 2005;11:406-12. doi:10.1097/01. ccx.000.017.6695.92883.87

[7] Rudski LG, Lai WW, Afilalo J, et al. Guidelines for the echocardiographic assessment of the right heart in adults: a report from the American Society of Echocardiography. Endorsed by the European Association of Echocardiography, a registered branch of the European Society of Cardiology, and the Canadian Society of Echocardiography. JAm Soc Echocardiogr 2010;23:685-713. doi:10.1016/j. echo.2010.05.010

[8] Thanakitcharu P, Charoenwut M, Siriwiwatanakul N. Inferior vena cava diameter and collapsibility index: a practical noninvasive evaluation of intravascular fluid volume in criticallyill patients. JMedAssocThailand = Chotmaihet thangphaet 2013;96 Suppl 3:S14-22.

[9] Wang WJ, Zhang XM, Wang Q, et al. [Amino-terminal pro-brain natriuretic peptide and brain natriuretic peptide measurements under various detection conditions in patients with chronic heart failure]. Zhonghua Xin Xue Guan Bing Za Zhi 2011;39:695-9.

[10] Lee SW, Song JH, Kim GA, Lim HJ, Kim MJ. Plasma brain natriuretic peptide concentration on assessment of hydration status in hemodialysis patient. Am J Kidney Dis 2003;41:125766. doi: 10.1016/s0272-6386(03)00358-5

[11] Crepaldi C, Lamas EI, Martino FK, et al. Bioimpedance and brain natriuretic peptide in peritoneal dialysis patients. Contrib Nephrol 2012;178:174-81. doi: 10.1159/000337849

[12] Tapolyai M, Faludi M, Réti V, et al. Volume estimation in dialysis patients: the concordance of brain-type natriuretic peptide measurements and bioimpedance values. International Symposium on Home Hemodialysis. Hemodial Int 2013;17:406-12. doi: 10.1111/hdi.12023

[13] Ferrada P, Anand RJ, Whelan J, et al. Qualitative assessment of the inferior vena cava: useful tool for the evaluation of fluid status in critically ill patients. Am Surg 2012;78:468-70.

[14] Zhang Z, Xu X, Ye S, Xu L. Ultrasonographic measurement of the respiratory variation in the inferior vena cava diameter is predictive of fluid responsiveness in critically ill patients: systematic review and meta-analysis. Ultrasound Med Biol 2014;40:845-53. doi:10.1016/j.ultrasmedbio.2013.12.010

[15] Dipti A, Soucy Z, Surana A, Chandra S. Role of inferior vena cava diameter in assessment of volume status: a meta-analysis. Am JEmerg Med 2012;30(8):1414-9.e1. doi: 10.1016/j. ajem.2011.10.017 
[16] Orso D, Paoli I, Piani T, Cilenti FL, Cristiani L, Guglielmo $\mathrm{N}$. Accuracy of ultrasonographic measurements of inferior vena cava to determine fluid responsiveness: A systematic review and meta-analysis. JInt Care Med 2020;35:354-63. doi: 10.1177/088.506.6617752308

[17] Long E, Oakley E, Duke T, Babl FE. Does respiratory variation in inferior vena cava diameter predict fluid responsiveness: A systematic review and meta-analysis. Shock2017;47:550-9. doi:10.1097/shk.000.000.0000000801

[18] Zhang X, Luan H, Zhu P, Feng J, Cui J, Zhao Z. Does ultrasonographic measurement of the inferior vena cava diameter correlate with central venous pressure in the assessment of intravascular volume in patients undergoing gastrointestinal surgery? Journal Surg Res2014;191:339-43. doi: 10.1016/j.jss.2014.04.043

[19] Muller L, Bobbia X, Toumi M, et al. Respiratory variations of inferior vena cava diameter to predict fluid responsiveness in spontaneously breathing patients with acute circulatory failure: need for a cautious use. Crit Care 2012;16:R188. doi:10.1186/cc11672

[20] Hebl V, Zakharova MY, Canoniero M, Duprez D, Garcia S. Correlation of natriuretic peptides and inferior vena cava size in patients with congestive heart failure. Vasc Health Risk Manag 2012;8:213-8. doi: 10.2147/vhrm.s30001

[21] Goonewardena SN, Gemignani A, Ronan A, et al. Comparison of hand-carried ultrasound assessment of the inferior vena cava and N-terminal pro-brain natriuretic peptide for predicting readmission after hospitalization for acute decompensated heart failure. JACC Cardiovasc Imaging 2008;1:595-601. doi: 10.1016/j.jcmg.2008.06.005

[22] Yavaşi Ö, Ünlüer EE, Kayayurt K, et al. Monitoring the response to treatment of acute heart failure patients by ultrasonographic inferior vena cava collapsibility index. Am J Emerg Med 2014;32:403-7. doi: 10.1016/j.ajem.2013.12.046 